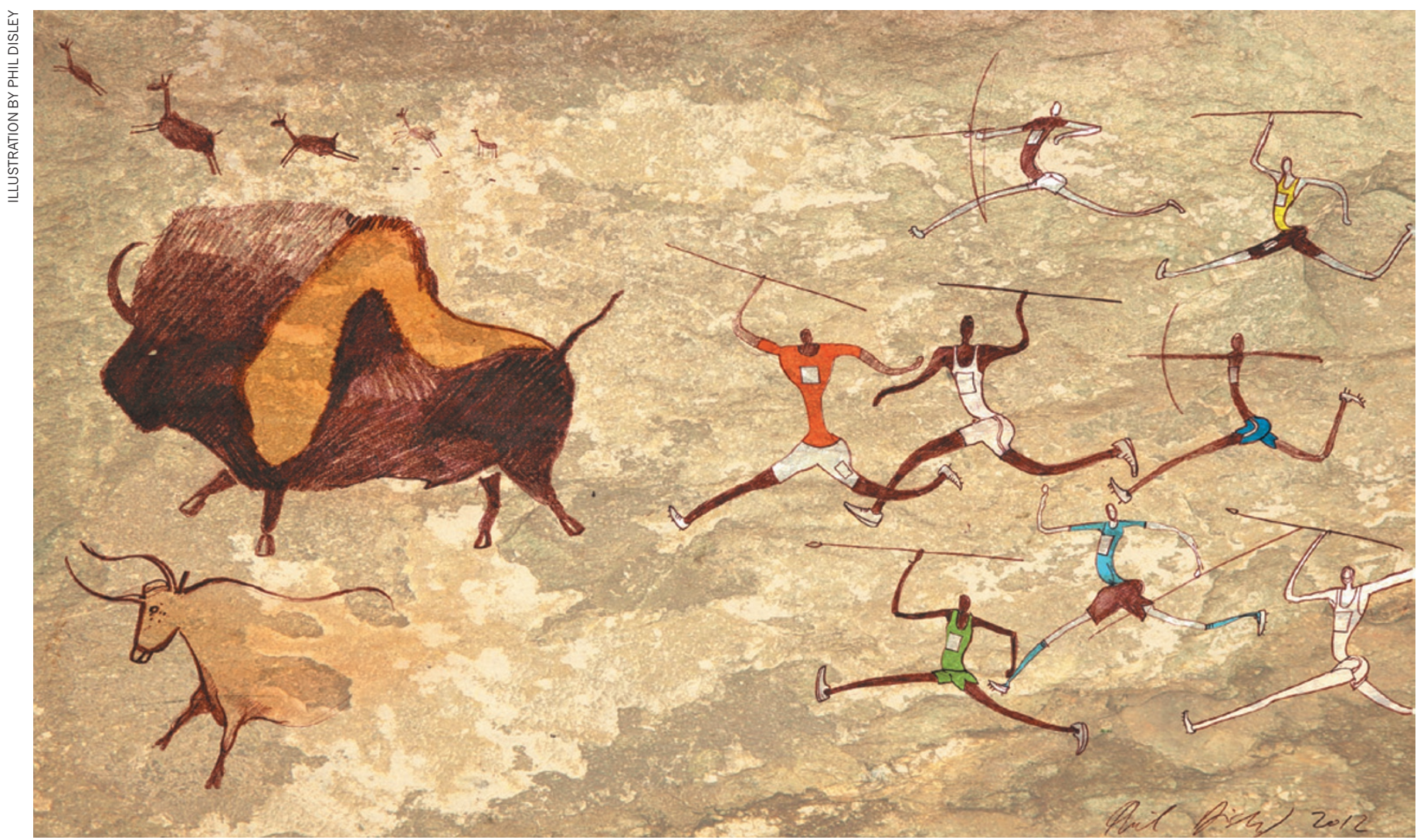

\title{
Run for your life
}

\section{Humans evolved to run. This helps to explain our athletic capacity and our susceptibility to modern diseases, argue Timothy Noakes and Michael Spedding.}

$\mathrm{T}$ The forthcoming Olympics in London will celebrate the performance capacity of humans and our remarkable ability to prepare our bodies and minds for specific tasks. But, at the same time as we are pushing our bodies to new limits in athleticism, we are experiencing unprecedented levels of relatively modern diseases such as obesity, diabetes and psychiatric and neurodegenerative disorders.

We, the authors, were both considering the modern paradox of elite athleticism and growing susceptibility to disease when we met at a sports conference in Glasgow, UK, in 2010. Noakes is a sports scientist who has run more than 70 marathons and ultramarathons. He was presenting data suggesting that humans' unmatched ability to dissipate heat when running, even when drinking sparingly, might have been a key element that enabled them to evolve from tree-living primates. Spedding, a pharmacologist presenting studies of how stress can increase the risk of psychiatric disorders, has run more than 100,000 kilometres and been a competitive athlete for more than 40 years. His brother,
Charlie, holds the English marathon record and won Olympic bronze in 1984 by ignoring drink stations at crucial stages in the Los Angeles marathon. We began exchanging e-mails. Eventually, that correspondence coalesced into the theory we outline here.

Over millions of years, humans evolved from tree-dwelling apes to become Homo sapiens, capable of elite athleticism ${ }^{1}$. Simply put, we evolved to run. While early hominins were undergoing intense skeletal and metabolic changes, major changes also occurred in their brains ${ }^{2}$. We propose that these changes have rendered us dependent on mental and physical exercise to maintain brain health. Exercise doesn't just help muscles - it activates our brains, particularly through one pathway that helps to increase the number of neuronal connections.

Most humans today do not live in an environment where they must exercise

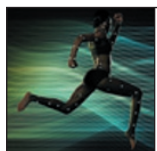

LET THE GAMES BEGIN Science and the Olympics nature.com/olympics 2012 regularly to chase down meat. For many, exercise is no longer an integral part of daily life, leading to a host of modern ailments.

"Most humans
today do not
live in an
environment
where they
must chase
down meat."

In short, we think that exercise is not just important for general health - it is essential to the molecular memory of who we are. Without it, we are at risk of being obese and diabetic, and of developing diseases linked to brain function, such as psychiatric disorders, dementia and even violent behaviour.

\section{COOL AND SWEATY}

The ancestors of modern humans were omnivorous apes with bodies that were more suited to living in trees than hunting in open habitats. Over the past few million years, the climate underwent dramatic shifts and Africa changed from a largely forested ecosystem to a more open savannah. Our ancestors, caught at the edge of the retreating forests, became less adapted for climbing 
trees. By 2 million years ago, they had evolved a skeleton that could support walking and running - partly so that they could hunt by pursuing individual animals for hours at a time ${ }^{1}$.

For more than 1 million years, there were no weapons other than stones or sharpened sticks. The best weapon was endurance. The predators had to outlast their prey, and so had many adaptations that enabled them to walk and run long distances, forcing their prey to gallop. Because four-legged animals cannot lose heat by panting and galloping at the same time, human hunters eventually drive their prey into heat stroke, so that the animal can be caught and killed with very simple weapons.

The ability of humans to dissipate heat comes from our lack of body hair and capacity to breathe through our mouths and to sweat at rates of up to 3 litres an hour, much more effective than panting. In a 3-hour hunt - or in a marathon - fit humans can safely lose up to $10 \%$ of their bodyweight ${ }^{3}$.

At the same time, the hunter needed planning and spatial navigation to follow prey for hours, coupled with social interactions so that groups could work together to isolate prey $^{4}$. Primitive hunter-gatherer societies have a uniquely human social structure with multiple interactions between non-family members, requiring advanced social skills ${ }^{4}$. Within a relatively short time, our ancestors' skeleton, brain, spatial tracking, communication and ability to dissipate heat shifted dramatically, allowing them to fill a very different niche. They developed longer legs, shorter toes, longer Achilles tendons, wider shoulders and a stronger gluteus maximus for running ${ }^{1}$. In addition, they evolved larger weight-bearing joints that could support long runs while avoiding too much damage.

It is likely that humans also have a much higher metabolic capacity than our ancestors did, measured by our ability to take up and use oxygen $\left(\mathrm{VO}_{2} \mathrm{max}\right)$. We are the only primates with the aerobic capacity to support long-distance running. It is otherwise restricted to migratory ungulates (horses, wildebeest) and social carnivores (hyenas, wolves). Humans evolved from animals with a low $\mathrm{VO}_{2}$ max to the modern endurance athlete's maximum capacity approaching 90 millilitres of oxygen per kilogram of body mass per minute.

Exercise remains central to our basal metabolism, even though it is no longer a core part of life for some human populations. For example, persistence hunting led to humans having great capacity to conserve sodium and retain water when either are in short supply ${ }^{3}$.

We think that one protein could have been central to these dramatic physiological changes, both mental and physical: brainderived neurotrophic factor (BDNF), which increases with exercise and play, and sculpts the developing brain (see 'Memory jog').

Although some research has yielded mixed results about the benefits of exercise for the brain ${ }^{5}$, the literature in general shows that regular exercise can have many cognitive benefits. Some research suggests that exercise has antidepressant effects, at least against mild depression, and may even offset Alzheimer's disease: an exercise programme has been shown to increase the volume of the brain's hippocampus. More data are needed to obtain a clearer picture of the effects of exercise on cognition. Ongoing clinical trials are testing the effects of exercise on Alzheimer's disease and schizophrenia.

\section{EXERCISE FOR LIFE}

We believe that at least some of these beneficial effects are mediated by BDNF. First, circulating BDNF is increased by exercise, partly by release from the nerve-muscle junction into the blood. In muscle, BDNF can increase protein synthesis and fat metabolism $^{5,6}$, which are key targets of exercise. Mice that lack BDNF become obese and there are strong inverse links between BDNF and type 2 diabetes $^{7}-$ a disease for which it is well known that exercise, with the appropriate dietary changes, is the best therapy allied to medication.

In the brain, BDNF increases neuronal connections and is crucial for some aspects of memory ${ }^{6}$. BDNF plays a part in the

\section{MEMORY JOG}

The beneficial effects of exercise on the body and brain are increasingly thought to be mediated by the brain protein BDNF.

\section{EXERCISE}

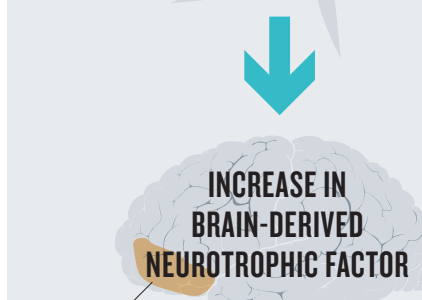

Ventromedial

prefrontal cortex

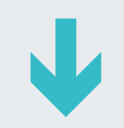

- Improves metabolism.

- Opposes neurodegenerative processes.

- Acts on spinal cord to reduce heart rate.

- Reduces rates of obesity and type 2

diabetes in mice.

- Increases connectivity between neurons.

- Triggers brain mitochondria to use oxygen

more efficiently.

- Has a role in psychiatric and

neurological disorders. hypothalamus, controlling body weight and energy homeostasis ${ }^{7}$. It also triggers the brain mitochondria - the powerhouses of the cell - to use oxygen more efficiently ${ }^{8}$, in a similar way to how exercise helps to increase overall $\mathrm{VO}_{2} \max$ in humans.

As humans needed more brain power to track prey, increases in BDNF may have helped to build up the hippocampus and prefrontal cortex - key brain areas that are involved in spatial mapping, decision-making and control of context, fear and emotions, including violence ${ }^{8-10}$. BDNF has a crucial role in the prefrontal cortex, a region that is also strongly associated with psychiatric disorders ${ }^{9}$. Not surprisingly, BDNF is reduced in hippocampal and cortical regions in models of stress and psychiatric disorders ${ }^{10}$, as well as in Alzheimer's disease. Putting it all together, we think that exercise increases BDNF in key areas of the brain, which, in turn, has physiological effects that help to protect humans from psychiatric problems, including violent behaviour.

Not all of the health benefits of exercise stem from the effects of BDNF alone, because diabetes and obesity are known to have other, multiple causes. Importantly, the modern diet - which is far removed from the whole foods our ancestors ate - can itself lead to obesity and inflammation, which counteract many of exercise's benefits.

A lack of exercise may have multiple, longterm damaging effects, particularly when coupled with a poor diet. Simple exercise programmes in schools and sports clubs, for example, are probably the most costeffective investments a society can make in its psychological and physical health. Exercise may be cheap, but the consequences of ignoring it are costly.

Timothy Noakes is professor of exercise and sports science at the University of Cape Town, Rondebosch 7701, South Africa. Michael Spedding is a pharmacologist and lives at Le Vésinet, near Paris, France. e-mails: timothy.noakes@uct.ac.za; spedves2@orange.fr

1. Bramble, D. M. \& Lieberman, D. E. Nature $\mathbf{4 3 2}$, 345-352 (2004).

2. Konopka, G. \& Geschwind, D. H. Neuron 68 , 231-244 (2010).

3. Noakes, T. Waterlogged: The Serious Problem of Overhydration in Endurance Sports (Human Kinetics, 2012).

4. Hill, K. R. et al. Science 331, 1286-1289 (2011).

5. Chalder, M. et al. Br. Med. J. 344, e2758 (2012).

6. Mattson, M. P. Ageing Res. Rev. 11, 347-352 (2012).

7. Noble, E. E., Billington, C. J., Kotz, C. M. \& Wang, C. Am. J. Physiol. Regul. Integr. Comp. Physiol. 300, R1053-R1069 (2011).

8. Markham, A. et al. Eur. J. Neurosci. 35, 366-374 (2012).

9. Agid, Y. et al. Nature Rev. Drug Discov. 6, 189-201 (2007).

10. Qi, H. et al. Neuropharmacology 56, 37-46 (2009).

M.S. declares competing financial interests; see go.nature.com/rhkkl5. 